\title{
The Sun-Earth Connection near Solar Minimum: Placing it into Context
}

\author{
Mario M. Bisi • Barbara J. Thompson • \\ Barbara A. Emery • Sarah E. Gibson · John Leibacher • \\ Lidia van Driel-Gesztelyi
}

Received: 14 November 2011 / Accepted: 14 November 2011 / Published online: 30 November 2011 (C) Springer Science+Business Media B.V. 2011

The Sun-Earth Connection near Solar Minimum Guest Editors: M.M. Bisi, B.A. Emery, and B.J. Thompson

M.M. Bisi $(\bowtie)$

Aberystwyth University, Aberystwyth, Wales, UK

e-mail: Mario.Bisi@aber.ac.uk

B.J. Thompson

NASA Goddard Space Flight Center, Greenbelt, MD, USA

e-mail: barbara.j.thompson@nasa.gov

B.A. Emery · S.E. Gibson

High Altitude Observatory, National Center for Atmospheric Research, 3080 Center Green, Boulder, CO 80301, USA

\section{B.A. Emery}

e-mail: emery@ucar.edu

S.E. Gibson

e-mail: sgibson@ucar.edu

\section{J. Leibacher}

National Solar Observatory, Tucson, AZ 85719, USA

e-mail: john.leibacher@gmail.com

\section{J. Leibacher}

Institut d'Astrophysique Spatiale, Orsay, France

L. van Driel-Gesztelyi

LESIA-Observatoire de Paris, CNRS, UPMC Univ. Paris 06, Univ. Paris-Diderot, Meudon Cedex, France

e-mail: Lidia.vanDriel@obspm.fr

L. van Driel-Gesztelyi

Mullard Space Science Lab., University College London, Holmbury St. Mary, RH5 6NT, UK

L. van Driel-Gesztelyi

Konkoly Observatory of the Hungarian Academy of Sciences, Budapest, Hungary 


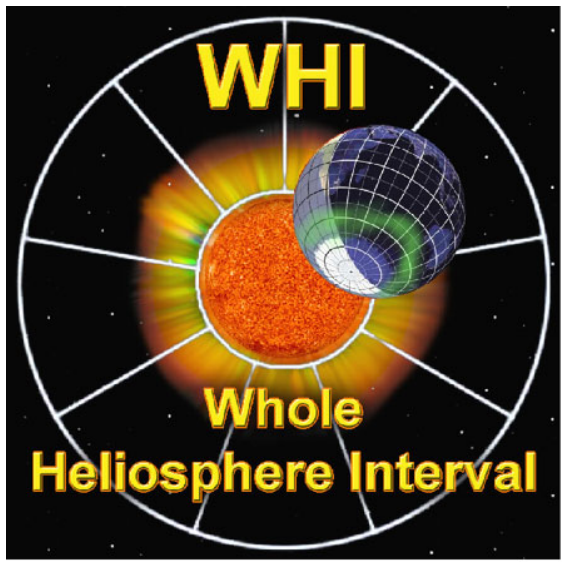

Solar minima represent times of low magnetic activity and simple heliospheres. They are thus excellent targets for interdisciplinary, systemwide studies of the origins of solar variability, and consequent impacts on planetary systems. The recent solar minimum extended longer and was "quieter" than any that we have observed in the Space Age, inspiring both scientific and public interest. A rich variety of satellite and ground-based observations, in conjunction with theoretical and numerical modeling advances, have allowed us to probe the peculiarities of this minimum as never before.

This Topical Issue (TI) of Solar Physics presents articles based on studies ranging around the solar minimum, and more specifically, studies of the Whole Heliosphere Interval (WHI). WHI was an internationally coordinated observation-and-modeling effort to characterize the three-dimensional (3D) heliosphere and the Sun-planet connectivity during solar minimum. WHI was the largest Coordinated Investigation Program (CIP) of the International Heliophysical Year (IHY) (2007 - 2009), which was inspired by the 50th Anniversary of the International Geophysical Year (IGY) (1957-1958) and the subsequent 50 years of space exploration. WHI, or Carrington Rotation 2068 (CR 2068), ran from 20 March 2008 to 16 April 2008 as viewed from Earth. This CR was thus the origin of the 3D structures and interactions studied, but the data collected came from a larger temporal range in order to trace the solar wind's outflow from the Sun right through the heliosphere including its interaction with the Earth and other planetary bodies. (See http://ihy2007.org/WHI for more information.)

In order to accomplish WHI's scientific goals of characterizing the 3D solar minimum heliosphere and tracing connections from Sun to Earth and beyond, observations were taken during the campaign that included both synoptic (ongoing) data and targeted campaigns. Connecting and interpreting these observations required the application of models and the coordination of the broad-ranging data. This was done via the WHI web site, WHI special sessions of international meetings (Spring and Fall 2008, and Spring 2010 AGU; 2009 IAU General Assembly in Rio de Janeiro, Brazil and the 2011 IAU Symposium 286 in Mendoza, Argentina). Moreover, two WHI workshops took place in Boulder, CO, USA. These workshops were primarily organized by Sarah Gibson (NCAR), Barbara Thompson (NASA), and David Webb (AFRL/Boston College), and the workshop venue and conference facilities were provided by the National Center for Atmospheric Research (NCAR) which is supported by the National Science Foundation (NSF).

The first Whole Heliosphere Interval Data and Modeling Assessment Workshop (WHIDMAW) was held 26-29 August 2008, during the 50th anniversary year of Parker's epochal paper on the formation of the solar wind which transformed our understanding of the environment in which the Earth is located. The goals of this first WHIDMAW were to gather data and model information, perform preliminary assessment and analysis, and connect and link the various data and models.

The second WHIDMAW was held 10-13 November 2009, in the year which marked the 400th anniversary of Galileo's sunspot observations and also the 150th anniversary of Carrington and Hodgson's independent observations of a white-light solar flare - the event from which we number Carrington rotations. This second WHIDMAW further promoted 
WHI data and modeling efforts. In particular, working groups were formed to consider the origins of solar variability, to compare data and models from Sun to Earth, to weigh the importance of magnetic boundary conditions, to establish the evolutionary context of CMEs and active regions, and to study solar irradiance and its implications for the Earth's atmosphere. It was at this second WHIDMAW that this TI was envisaged and the processes put in place to get the TI underway.

This TI includes scientific results of the observations, measurements, and modeling techniques that are used to interpret the WHI data, including all of the regimes that play a role in solar minimum heliophysics. These regimes include:

- sub-photospheric structures;

- the solar photosphere, chromosphere, and corona;

- the inner and outer heliosphere;

- Earth's magnetopause and bow shock;

- Earth's radiation belts and plasmasphere; and

- the thermosphere and ionosphere.

Additionally, the TI includes contributions that are linked to and/or are related to WHI but not necessarily centered on WHI itself. The majority cover the present solar minimum, and also how this minimum compares and relates to past minima. One of the earliest findings of WHI was that, although initial forecasts predicted solar minimum to occur in late 2006/early 2007, the Sun during WHI in Spring 2008 was still exhibiting structure consistent with the declining phase of Solar Cycle 23 despite sunspot numbers lower than the last minimum. This spurred the formation of the International Astronomical Union Division II Working Group on Comparative Solar Minima (see http://ihy2007.org/IAUWG/WEBPAGES/IAUWG.shtml for further details).

As a result of the depth, length, and complexity of the current solar minimum, one solar rotation is not enough to characterize it. Therefore, two additional Carrington rotations were identified - WHI 2 and WHI 3 - that, along with WHI, may be considered to span the current extended solar minimum. These are, respectively, CR 2078 (17 December 2008 - 12 January 2009 when sunspots reached a 13-month minimum) and CR 2085 (26 June 2009-22 July 2009 when solar-wind parameters were at their lowest, and which had continuous SOHO contact and solar eclipse data/modeling). These rotations provide additional focus time periods that enable intercomparison between analyses in the TI, although studies were not limited to these three WHI rotations.

Finally, we would like to thank the authors for the articles from those not able to attend the WHIDMAWs in 2008 or 2009 as well as the articles from those in attendance, and to convey an extra-special thanks to the team of highly qualified referees who worked extremely hard in ensuring that the articles in this TI of Solar Physics on "The Sun-Earth Connection near Solar Minimum: Placing it into Context" are of outstanding scientific quality. We are very happy with the articles and the scope of the work covered in this TI, and we are pleased that they collectively cover many measurements, observations, and models of the current solar minimum, as well as comparisons to previous minima. They represent the full spectrum of heliophysical research, all the way from below the Sun's surface to well beyond 1 AU. 